\title{
Surgical recovery of sperm in non-obstructive azoospermia
}

\section{Tomomoto Ishikawa}

The development of intracytoplasmic sperm injection (ICSI) opened a new era in the field of assisted reproduction and revolutionized the assisted reproductive technology protocols for couples with male factor infertility. Fertilisation and pregnancies can be achieved with spermatozoa recovered not only from the ejaculate but also from the seminiferous tubules. The most common methods for retrieving testicular sperm in non-obstructive azoospermia (NOA) are testicular sperm aspiration (TESA: needle/fine needle aspiration) and open testicular biopsy (testicular sperm extraction: TESE). The optimal technique for sperm extraction should be minimally invasive and avoid destruction of testicular function, without compromising the chance to retrieve adequate numbers of spermatozoa to perform ICSI. Microdissection TESE (micro-TESE), performed with an operative microscope, is widely considered to be the best method for sperm retrieval in NOA, as larger and opaque tubules, presumably with active spermatogenesis, can be directly identified, resulting in higher spermatozoa retrieval rates with minimal tissue loss and low postoperative complications. Micro-TESE, in combination with ICSI, is applicable in all cases of NOA, including Klinefelter syndrome (KS). The outcomes of surgical sperm retrieval, primarily in NOA patients with elevated serum follicle-stimulating hormone (FSH) (NOA including KS patients), are reviewed along with the phenotypic features. The predictive factors for surgical sperm retrieval and outcomes of treatment were analysed. Finally, the shortand long-term complications in micro-TESE in both 46XY males with NOA and KS patients are considered.

Asian Journal of Andrology (2012) 14, 109-115; doi:10.1038/aja.2011.61; published online 28 November 2011

Keywords: azoospermia; micro-TESE; sperm retrieval; TESE

\section{SURGICAL SPERM RETRIEVAL}

Prior to the availability of assisted reproductive technologies, the use of donor sperm was the only option offering a realistic chance of conception for couples affected by azoospermia.

The approach to azoospermic patients has changed significantly with the introduction of sperm retrieval techniques and assisted reproductive technologies, especially intracytoplasmic sperm injection (ICSI). In addition to improving pregnancy rates using sperm from ejaculated semen, ICSI has provided new possibilities for achieving pregnancy with sperm retrieved from the epididymis or testis.

Azoospermia, defined as the absence of spermatozoa in the ejaculate after the assessment of centrifuged semen on at least two occasions, is observed in $1 \%$ of the general population and in $10-15 \%$ of infertile men. $^{1,2}$

Surgical sperm retrieval techniques are applied in azoospermia cases of either obstructive or non-obstructive aetiology. Remarkably, the first pregnancy using epididymal sperm and standard in vitro fertilisation was achieved in $1985,{ }^{3}$ and the first pregnancies after fertilisation by ICSI with testicular sperm from men with obstructive azoospermia (OA) were reported in 1993. ${ }^{4}$

Percutaneous epididymal sperm aspiration, microsurgical epididymal sperm aspiration and testicular sperm aspiration (TESA) are used for OA cases, while TESA and testicular sperm extraction (TESE) are applied in non-obstructive azoospermia (NOA) cases.
NOA

NOA affects $10 \%$ of infertile men and is diagnosed in $60 \%$ of azoospermic men. ${ }^{2}$ Aetiologies for testicular failure include genetic disorders, such as sexual chromosomal abnormalities, translocations and microdeletions of the Y chromosome, cryptorchidism, testicular torsion, radiation and toxins. ${ }^{2,5-7}$

It has been shown that mature spermatozoa can be found in only part of the testes of NOA patients. Testicular spermatozoa can be retrieved from some NOA men despite the absence of ejaculated spermatozoa in their semen because of the existence of isolated foci of active spermatogenesis.

In examining the testes of infertile men, Levin ${ }^{8}$ found a mixed histological pattern of germinal cell aplasia and minute focal spermatogenesis. A similar histology has been observed with the side-byside presence of different patterns of focal spermatogenesis and Sertoli cell-only syndrome (SCO) in NOA men., ${ }^{9,10}$ Therefore, the performance of multiple focal testicular sperm retrieval has been recommended to ensure the presence of sperm in testicular samples. ${ }^{11}$

\section{SPERM RETRIEVAL TECHNIQUES IN NOA}

Fine needle aspiration (FNA) and TESA

The most common methods for retrieving testicular sperm are TESA (needle/fine needle aspiration) and open testicular biopsy (TESE).

Lewin et al. ${ }^{12}$ successfully performed testicular FNA to retrieve sperm from a patient with maturation arrest (MA) and elevated

Department of Urology, Ishikawa Hospital, Himeji 6710221, Japan 
gonadotropins. Testicular needle aspiration was initially used for diagnostic purposes and later to establish the likelihood of sperm retrieval for testicular ICSI cases on the day of oocyte retrieval. ${ }^{13,14}$ To avoid postoperative testicular damage, a needle biopsy with a biopsy gun ${ }^{15-17}$ and needle aspiration ${ }^{18}$ have been utilized. Different techniques have been described, with variations in the needle diameter and the number of testicular punctures. ${ }^{19}$ Regarding the needle diameter for FNA, Rosenlund et al. ${ }^{20}$ concluded that 19-gauge needles are better than 21-gauge needles. Large needle percutaneous aspiration biopsy showed good results in a descriptive study (60\% sperm retrieval rate (SRR)), irrespective of testicular volume. ${ }^{21}$ However, another study showed that TESE yielded a significantly higher SRR compared with FNA, despite the use of an 18 -gauge needle $(62.1 \%$ vs. $24.1 \%){ }^{22}$

FNA is usually performed in 1-6 different locations, in the centre of the testis and in the upper and lower poles, with the aim of aspirating testicular tissue from the depth of the testis. Upon stabilizing the testis and stretching the scrotal skin, the needle is inserted into the anterior face of the testis, and negative pressure is applied. While maintaining negative pressure, the needle is partially withdrawn and inserted again at different angles.

FNA has also been proposed as a mapping technique for performing multiple punctures prior to TESE. ${ }^{14}$ FNA mapping has shown a high cytohistological correlation with multiple biopsies (92\%). ${ }^{23}$

The main advantages of the FNA and TESA techniques are their simplicity, low cost and minimal invasiveness and that they produce less post-operative pain compared with multiple TESE under local anaesthesia. ${ }^{24}$ Moreover, it has been suggested that FNA can increase the chance of finding a site of active spermatogenesis by reaching deeper testicular sites. ${ }^{24}$ However, several controlled studies have shown a significantly lower SRR compared with simple or multiple TESE. $^{20,24}$ In addition, a disadvantage of FNA and TESA is that frequently there are no supernumerary spermatozoa to cryopreserve because of the limited number retrieved. ${ }^{25}$ In fact, a prospective study reported a significantly smaller number of frozen straws per patient compared with multiple TESE. ${ }^{11}$ The authors also showed that a significantly lower motile SRR was obtained when only FNA was performed, compared with conventional TESE.

\section{Simple TESE and multiple TESE}

In general, for multiple TESE, the tunica albuginea is incised transversely at several locations of the centre and upper and lower poles of each testis. The testis is then gently squeezed, and the protruding tissues are excised, each weighing approximately 50-100 mg.

Several TESE procedures for patients with NOA have also been reported. Simple TESE (open single biopsy) is the main procedure for NOA, on the basis of the assumption that multifocal distribution of spermatogenesis throughout the entire testis is present in NOA patients. A single incision and large volume of tissue result in a high retrieval rate but also result in the increased loss of testicle volume. Other researchers strongly recommend multiple TESE. ${ }^{26}$ It is reasonable to assume that more testicular tissue increases the prospects for the identification of spermatozoa. Indeed, multiple TESE is more effective than needle biopsy for the retrieval of spermatozoa in patients with NOA. ${ }^{27,28}$ Significantly larger numbers of sperm cells, locations with spermatogenesis per testis and motile sperm cells per testis have been reported following conventional TESE compared with FNA. ${ }^{11}$

The most appropriate number of biopsies to be taken remains controversial. Single testicular biopsy has been refuted by several studies that found a patchy distribution of regions with minimal spermatogenesis throughout the testis. ${ }^{11,29}$ Therefore, taking multiple samples from different sites of the testis could increase the chance of finding a focus of active sperm production. In addition, it has been shown that the number of biopsies required to retrieve motile sperm is significantly higher in cases of MA and SCO, compared with patients with hypospermatogenesis. ${ }^{29}$

In addition, the multiple biopsy approach enables a significantly higher SRR compared with single biopsy (49\% vs. 37.5\%). ${ }^{30}$ Taking multiple biopsies could also increase the chance of finding motile sperm, as it was shown that in $35 \%$ of the cases, only non-motile spermatozoa were found in one location and motile spermatozoa were found in others. ${ }^{11}$

Regarding the location of the biopsy, Hauser et al. ${ }^{11}$ found no advantage of any particular site of the testicle after performing biopsies on the upper pole, midline and proximal pole, whereas Witt et al. ${ }^{31}$ concluded that the midline portion of the testis enabled the highest SRR.

Large resections can result in postoperative testicular damage. Multiple TESE may result in devascularisation because of the limited blood supply to the testicle flowing beneath the tunica albuginea before it penetrates into the testicular parenchyma. Multiple TESE should be performed cautiously because these interventions could injure enough of the blood supply to risk testicular devascularisation and result in permanent androgen deficiency.

\section{Micro-TESE}

The optimal technique for sperm extraction would be minimally invasive and avoid destruction of testicular function, without compromising the chance to retrieve adequate numbers of spermatozoa to perform ISCI.

Schlegel $^{32}$ have combined simple TESE and multiple TESE techniques with the assistance of an operating microscope and have successfully developed a technique known as micro-TESE.

Micro-TESE is an advanced version of TESE that applies microsurgical techniques to identify individual seminiferous tubules that are more likely to contain active spermatogenesis. ${ }^{32}$

It has also been noted that micro-TESE performed while observing the seminiferous tubules under an operating microscope could minimize the damage to testicular tissue while maximizing sperm recovery compared with simple or multiple TESE. ${ }^{32}$ Micro-TESE has also been shown to be more successful in sperm retrieval than a single biopsy or multiple random biopsies. ${ }^{33,34}$ In addition, micro-TESE improves the yield of spermatozoa per biopsy, results in less tissue removal (and loss of testicle) and allows for the identification of blood vessels within the testicle, minimizing the risk of vascular injury and loss of other areas of the testis. ${ }^{32}$ Thus, micro-TESE is based on the principle of identifying the most advanced pattern, though not necessarily the predominant pattern, of spermatogenesis in the testis.

In cases of NOA, the sperm retrieval procedure is often extensive, involving general anaesthesia and micro-TESE until sufficient spermatozoa are extracted for ICSI, as described below in Schlegel's method.

A midline incision is made in the scrotum, the tunica vaginalis is opened, and the testis, which is covered with the tunica albuginea, is visualized. The remainder of the procedure is performed under an operative microscope. After the tunica albuginea has been opened, direct examination of the testicular parenchyma is performed at $\times 15$ magnification (Figure 1). The tunica albuginea is opened on an equatorial plane because an antimesenteric incision increases the risk of testicular devascularisation and may adversely affect access to the seminiferous tubules. A transverse incision appears to reduce the 


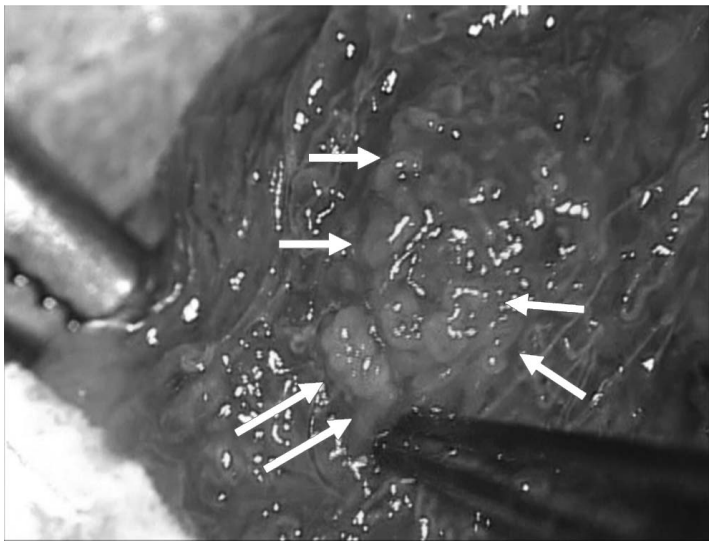

Figure 1 Focal spermatogenesis could be identified in dilated and opaque seminiferous tubules surrounded by shrunken tubules or fibrous tissue in a Klinefelter syndrome (KS) patient.

risk of vascular injury because the testicular artery enters the testis posteriorly beneath the epididymis at the mid-pole, continues inferiorly to the lower pole, and then flows superiorly along the anterior surface, where it gives rise to transverse branches that supply the parenchyma. The examination includes as much of the testicular parenchyma as possible. Small samples $(5-10 \mathrm{mg})$ are excised from the tubules. If no spermatozoa are identified in the initial sample, then subsequent samples are taken from the same testis and, if needed, from the contralateral testis. Dissection is performed through all regions of testicular tissue, preserving the testicular blood supply. The procedure is terminated when spermatozoa are retrieved or when further dissection is thought likely to jeopardize the testicular blood supply. ${ }^{35}$ Most of the studies have defined success as the recovery of at least one spermatozoa; however, others also consider the morphology and motility of the retrieved sperm as relevant parameters. In general, we define the success of sperm retrieval as the recovery of at least one testicular spermatozoon.

\section{SRR BY MICRO-TESE}

The outcomes of micro-TESE have been reported (Table 1). ${ }^{32-40}$ In 1999, the first report on this technique compared 22 patients who underwent standard multiple biopsies with a group of 27 men who underwent micro-TESE. The author described a significant improvement in the SRR when micro-TESE had been performed $(63 \%$ vs. $45 \%) .{ }^{32}$ A comparative study that included 116 men found a significantly higher SRR with the addition of optical magnification compared with conventional TESE ( $47 \%$ vs. $30 \%)$, indicating the efficacy of micro-TESE for sperm retrieval. ${ }^{36}$ To date, the largest study series on micro-TESE reported the results of 792 procedures, which achieved a SRR of $60 \% .{ }^{39}$ Micro-TESE is a highly effective technique to obtain sperm in the setting of NOA but requires a learning curve. We reported, regarding the learning curves, that the SRRs for NOA patients in the middle 50 cases (44\%) and the last 50 cases (48\%) were significantly higher than for the first 50 cases $(32 \%){ }^{40}$ The SRR of micro-TESE is strongly influenced by the surgeon's case volume, as a considerable number is necessary to reach an optimal plateau level for the SRR in this microscopic surgery. ${ }^{40}$ The outcome of SRR may depend on factors other than surgical technique, such as embryologists' learning curves.

Tsujimura et al. ${ }^{34}$ reported the SRR by micro-TESE compared with that by conventional TESE for each pattern of testicular histology in patients with NOA; the SRR by micro-TESE for patients with SCO was $22.5 \%$, whereas that by conventional TESE was $13 \%$. Furthermore, the SRR by micro-TESE for patients with hypospermatogenesis was $100 \%$, while that by conventional TESE was $76.9 \%$. Okada et al. ${ }^{33}$ also reported reasonable results, showing that the SRR by micro-TESE for hypospermatogenesis was $100 \%$. They further reported that the SRRs by micro-TESE for MA and SCO were $75 \%$ and $33.9 \%$, respectively, while those by conventional TESE were $37.5 \%$ and $6.3 \%$, respectively. Ramasamy et al. ${ }^{35}$ reported the outcomes of 460 patients with NOA treated with micro-TESE. In that report, the SRR by microTESE was $57 \%$, whereas that by conventional TESE was $32 \%$. They reported excellent SRRs of $81 \%, 44 \%$ and $41 \%$ for hypospermatogenesis, MA and SCO, respectively.

Micro-TESE is also effective for patients in whom conventional TESE has failed. By conventional TESE, spermatozoa may not be retrieved in patients who previously underwent conventional TESE because of testicular damage caused by the first TESE. ${ }^{30,41,42}$ Tsujimura et al..$^{43}$ found comparable results in patients undergoing primary micro-TESE ( $45.7 \%$ vs. $44 \%)$ and after a failed conventional TESE. Ramasamy and Schlegel ${ }^{44}$ also reported a lack of threshold for the number of previous negative biopsies before undergoing microTESE, although the SRR was significantly lower in patients who underwent three or four biopsies, compared with one or two biopsies $(23 \%$ vs. $51 \%$ ). Thus, the possibility of spermatozoa retrieval by salvage micro-TESE remains, regardless of the previous failure of other TESE procedures.

\section{MICRO-TESE FOR KLINEFELTER SYNDROME (KS)}

KS is the most common sex-chromosome disorder among infertile males, with a prevalence of 1 in $660 \mathrm{men}^{45}$ and is a frequent cause of hypogonadism and infertility. KS may present with delayed sexual maturation, increased height, learning and behavioural difficulties, varicosities, obesity, diabetes, leukaemia, increased likelihood of extragonadal germ cell tumours, and breast cancer (20-fold higher than in normal males). Approximately $80 \%$ of individuals with KS have a uniform 47,XXY karyotype (non-mosaic type), and the other

Table 1 Comparison of the sperm retrieval rate (SRR) between conventional and micro- testicular sperm extraction (TESE)

\begin{tabular}{|c|c|c|c|c|}
\hline Year & Author & Case (n) & SRR by conventional TESE (\%) & SRR by micro-TESE (\%) \\
\hline 1999 & Schlegel $^{32}$ & 27 & 45 & 63 \\
\hline 2000 & Amer et al. ${ }^{36}$ & 100 & 30 & 47 \\
\hline 2002 & Okada et al. ${ }^{33}$ & 74 & 16.7 & 44.6 \\
\hline 2002 & Tsujimura et al. ${ }^{34}$ & 56 & 35.1 & 42.9 \\
\hline 2004 & Tsujimura et al. ${ }^{37}$ & 180 & - & 44.4 \\
\hline 2005 & Ramasamy et al. 35 & 460 & 32 & 57 \\
\hline 2005 & Mulhall et al.. & 48 & 50 & 45 \\
\hline 2009 & Ramasamy et al. ${ }^{39}$ & 792 & - & 60 \\
\hline 2010 & Ishikawa et al. ${ }^{40}$ & 150 & - & $42(32,44,48)$ \\
\hline
\end{tabular}


$20 \%$ have either $47, \mathrm{XXY} / 46, \mathrm{XY}$ mosaics or have higher-grade aneuploidy of the sex chromosomes. ${ }^{45}$ Reproductive hormone studies usually demonstrate decreased serum testosterone $(\mathrm{T})$ and elevated luteinizing hormone (LH) and follicle-stimulating hormone (FSH) levels. The histological pattern is one of SCO and often with areas showing sclerosis and hyalinisation of the seminiferous tubules.

A case of non-mosaic KS successfully treated by TESE was reported in $1996 .{ }^{29}$ Tournaye et al. ${ }^{46}$ also reported successful spermatozoa retrieval in four of nine KS patients $(44.4 \%)$ by conventional TESE in 1996. Reubinoff et al. ${ }^{47}$ reported successful spermatozoa retrieval in four of seven KS patients (57.1\%) by FNA in 1998.

Several studies have been conducted with micro-TESE in patients with KS. ${ }^{48-50}$ Schiff et al. ${ }^{49}$ reported an impressive SRR (69.0\%); however, these cases of KS included mosaic individuals. We reported an SRR of $52.4 \%$ of 21 patients with non-mosaic KS by micro-TESE. ${ }^{40}$ It has been reported that the patient age in successful TESE for cases of $\mathrm{KS}$ is significantly younger than it is in failed cases. ${ }^{48,51,52}$

In all patients in whom micro-TESE was successful, we could identify focal spermatogenesis in dilated and opaque seminiferous tubules that were surrounded by shrunken tubules or fibrous tissue (Figure 1). Micro-TESE is particularly helpful for successful sperm retrieval in KS cases. In NOA, the absence of uniformity in the testicular tissue is critical to the success and rationale for using micro-TESE. If a better or the best portion can be identified under the microscope, then there is an increased chance of retrieving testicular sperm.

Recently, Ramasamy et al. ${ }^{53}$ analysed the influence of preoperative hormonal therapy on the SRR for KS patients. They concluded that patients who required medical therapy and who responded to that treatment, with a resultant $\mathrm{T}$ level of $250 \mathrm{ng} \mathrm{dl}^{-1}\left(8.7 \mathrm{nmol} \mathrm{l}^{-1}\right)$ or higher, had a higher SRR than men in whom the post-treatment $\mathrm{T}$ level was less than $250 \mathrm{ng} \mathrm{dl}^{-1}\left(8.7 \mathrm{nmol} \mathrm{l}^{-1}\right)(77 \%$ vs. $55 \%) .^{53}$

In patients already receiving exogenous $\mathrm{T}$ treatment, it is recommended that this treatment be discontinued, for example, for at least 6 months before micro-TESE, in order to allow for an increase in endogenous gonadotropin levels and to reactivate spermatogenesis in any tubules that may respond. However, this strategy is not supported by solid evidence, and the cessation of $\mathrm{T}$ treatment may result in troubling symptomatic hypogonadism in many patients.

\section{PREDICTORS FOR SPERM RETRIEVAL}

Micro-TESE with subsequent assisted fertilisation by ICSI is now a popular approach in the management of couples with NOA. However, given the treatment demands on both partners and the associated emotional and financial burdens, it would be beneficial to be able to predict the success of sperm retrieval using non-invasive parameters before the attempted procedure. Any proposed prognostic parameter for successful sperm retrieval ought to be sufficiently robust as to meaningfully inform the couples' decision to proceed, given that alternative strategies are limited to donor insemination, adoption or childlessness.

It has been reported that there is no statistically significant difference in testicular volume between NOA patients in whom sperm could be retrieved and NOA patients in whom sperm could not be retrieved. ${ }^{9}$ In addition, no lower limit of testicular volume for the absence of spermatozoa has been identified. In comparing the SRR between conventional TESE and micro-TESE, a retrospective study found the latter to be only superior in men with a testicular volume less than $10 \mathrm{ml}$ (42\% vs. $27 \%){ }^{38}$

In recent years, the addition of non-invasive examinations such as colour Doppler ultrasound to guide FNA or TESE in the identification of regions with higher vascularisation that exhibit active spermatogenesis has been assessed. ${ }^{54}$ In addition, high-resolution colour Doppler ultrasound, performed prior to TESE and focused on the evaluation of tissue perfusion, has recently revealed a positive correlation with the quantity and quality of the isolated sperm. ${ }^{55}$ Hence, these techniques could reduce the number of biopsies required to retrieve sperm, thus minimizing testicular damage. Nevertheless, active spermatogenesis regions could not be predicted in general, so more studies are required to establish the added value of these non-invasive tests.

Many studies have shown a relationship between testicular histopathological findings and testicular sperm retrieval by TESE. ${ }^{56-58}$ Histological findings are generally the most useful predictive factors for successful TESE. However, diagnostic testicular biopsy may cause inflammatory changes, haematoma, parenchymal fibrosis or permanent devascularisation of the testes. ${ }^{41}$ Other factors that have shown an association with the probability of recovering sperm include a history of orchitis, testicular volume, age at orchidopexy for cryptorchidism, KS and azoospermia factor (AZF) microdeletions. ${ }^{59-62}$ In general, the SRR of ex-cryptorchid men by TESE is higher than that of non-cryptorchid men. Additionally, the SRR of KS patients by TESE is higher than that of 46XY patients in NOA. In addition, the SRR with AZFa and b complete microdeletion is $0 \%$.

An important preoperative serum parameter studied in the first years of TESE was FSH. In general, the serum concentration of FSH is inversely correlated with the impairment of spermatogenesis. Recent studies using multiple TESE techniques have shown that elevated FSH levels have been associated with a low probability for the retrieval of spermatozoa in men. ${ }^{63}$ Therefore, FSH may predict the presence of sperm at random biopsy using conventional TESE techniques. ${ }^{64-67}$ Although FSH reflects the predominant pattern of spermatogenesis, it may not reflect isolated areas of spermatogenesis within the testis. Serum FSH concentration is not related to the more advanced stages of spermatogenesis. ${ }^{68}$ The relationship between FSH and the presence of any spermatogenesis is not straightforward in men with NOA, including men with KS. Serum FSH level has a poor predictive value for successful micro-TESE. Ramasamy et al. ${ }^{39}$ showed that sperm retrieval was higher in NOA men with an FSH level $>15 \mathrm{IU} \mathrm{ml}^{-1}$ than in men with an FSH level $<15 \mathrm{IU} \mathrm{ml}^{-1}$. Also, SRRs were maintained even when the FSH value was markedly elevated. A lower FSH level may be a reflection of the larger number of Sertoli cells in a larger testis, providing more control feedback to suppress FSH production. The excellent findings with higher FSH levels may reflect the sensitivity of microdissection in finding small areas of sperm production. These findings further illustrate that FSH is not able to resolve spermatogenesis on an individual tubule level, and therefore, it should not be used as a predictor of sperm recovery.

Several studies have correlated the inhibin B level with spermatogenetic activity, as determined by histological examination of the testis. ${ }^{69,70}$ Inhibin B has also been found to be slightly more sensitive than FSH as an index of spermatogenic status. However, it was concluded that the inhibin B level alone, or in combination with the serum FSH level, fails to predict the successful outcome of TESE for patients with NOA. ${ }^{71}$ The predictive value of inhibin $\mathrm{B}$ is not considered to be sufficient to exclude or include patients for TESE. ${ }^{72,73}$

However, in some circumstances, testis size and FSH have been shown to predict successful micro-TESE. Ramasamy et al. ${ }^{74}$ retrospectively analysed the records of 126 men with NOA who underwent one successful microdissection testicular sperm extraction attempt. They showed that men with a successful repeat attempt had a lower FSH level and larger testicular volume at the repeat procedure compared with men with a failed repeat attempt. ${ }^{74}$ 
Age-related changes in human testes generally include reduced testicular size, impaired spermatogenesis, reduced tubule length, increased thickness of tubule boundary tissue, sclerosis, focal mononuclear orchitis, dilatation of the rete testis and an increased incidence of azoospermia. ${ }^{75}$ The aging process may worsen seminiferous conditions, even in patients with NOA. Okada et al. ${ }^{48}$ reported that they succeeded in obtaining spermatozoa in 26 of 51 non-mosaic KS patients and that the levels of LH, FSH and T, and testicular volume did not differ between patient groups, which were defined by success and failure. The median ages for successful and failed testicular sperm extraction were 31 and 38 years, respectively. The percentage of successful recovery of spermatozoa decreased after the age of 35 years old. It has also been reported that the patient age in successful TESE for cases of KS is significantly younger than that in failed cases. ${ }^{51,52} \mathrm{Koga}$ et al. ${ }^{50}$ showed that testicular spermatozoa were retrieved successfully in 13 (50\%) of 26 non-mosaic KS patients, although they did not find any factor predictive of successful testicular sperm retrieval by microTESE.

We have been able to retrieve testicular spermatozoa in 27 (54\%) of 50 non-mosaic KS patients thus far; however, we have not found a predictive factor retrospectively.

One must conclude that, at the present time, there are no absolute predictors of sperm yield for micro-TESE for NOA including KS, other than profound genetic lesions (AZFa or AZFb).

Ramasamy et al. ${ }^{76}$ concluded regarding operation time that the chance of sperm retrieval during micro-TESE is best during the first $2 \mathrm{~h}$ of the operation. However, they also showed that sperm were still found in up to $37 \%$ of men who required greater than $4 \mathrm{~h}$ of microdissection. $^{76}$

Further research should focus on the added value of non-invasive techniques, such as ultrasound, to identify the most likely areas from which to retrieve spermatozoa.

\section{POSTOPERATIVE COMPLICATIONS}

The testicular blood supply derives from the internal spermatic artery and cremasteric and vassal arteries, which penetrate the tunica albuginea and septa. ${ }^{77}$ Given testicular anatomic considerations, multiple testicular biopsy has been suspected to increase the risk of testicular damage caused by the interruption of branches of the testicular artery $^{41}$ or by pressure atrophy from intratesticular swelling and haematoma. ${ }^{78}$ Testicular damage secondary to surgery is either the consequence of interference with the vascular supply to the seminiferous tubules or increased intratesticular pressure secondary to bleeding within the tunica albuginea. ${ }^{78}$ The development of intratesticular haematoma has been observed in up to $80 \%$ of patients undergoing TESE with single or multiple biopsies, based on ultrasounds performed 3 months after surgery. ${ }^{41}$

Harrington et al. ${ }^{79}$ reported intratesticular bleeding in $29 \%$ of cases after TESE and in 7\% after FNA. Several studies have documented a lower incidence of haemorrhagic complications following microTESE compared with the conventional technique. ${ }^{32,33}$

Regarding fibrosis, multiple studies have shown ultrasonographic changes after TESE, which have been attributed to the development of scar tissue. ${ }^{36,41,77}$ Using ultrasonography, Schlegel and $\mathrm{Su}^{41}$ reported the incidence of testicular scars, impaired blood flow and devascularisation in subjects with NOA 3 months after open testicular biopsy. At 3 months after TESE, $82 \%$ of evaluated patients had ultrasonographic abnormalities in the testis, suggesting resolving inflammation or haematoma at the biopsy site. ${ }^{41}$

The removal of a lesser amount of testicular tissue with microTESE, compared with conventional TESE, could reduce the risk of complications. ${ }^{33}$ A comparative study based on testicular ultrasonography within 6 months of TESE showed that micro-TESE was less invasive than conventional TESE. ${ }^{36}$ Similar results were found in another study, with focal echogenic lesions in the TESE group compared with none in the micro-TESE group. ${ }^{33}$ In a large-scale study of 435 patients with NOA in whom micro-TESE or conventional TESE was performed, ultrasound findings showed fewer acute and chronic changes in the microdissection group than in the conventional group. ${ }^{35}$

Even micro-TESE may be expected to increase the risk of testicular damage caused by the interruption of branches of the testicular artery, pressure atrophy from intratesticular swelling, or increases in peritubular scar tissue that may affect Leydig cell as well as germ cell numbers. This technique was developed to minimize unnecessary damage to the testis and to improve sperm recovery.

Although numerous studies have compared conventional versus micro-TESE, ${ }^{33,34,36}$ there are few large, comprehensive studies analysing hormonal changes after micro-TESE. The most important advantage of micro-TESE is that meticulous haemostasis can be achieved under clear magnified vision. Ramasamy et al. ${ }^{35}$ reported that for NOA patients, the initial decrease in the $\mathrm{T}$ level was followed by a return to $95 \%$ of the pre-micro-TESE $\mathrm{T}$ levels after 18 months. In $\mathrm{KS}$, but not 46XY males, with NOA, a decrease in serum T levels after micro-TESE was noted. A recent study on micro-TESE for NOA also reported no change in T level after the procedure. ${ }^{80}$

Although micro-TESE reduces the amount of tissue required to retrieve spermatozoa, this technique is not exempt from complications, such as fibrosis and hypogonadism. In addition, especially in KS patients, data on hormonal changes after micro-TESE are lacking. Hypogonadism in KS is relative rather than absolute, and has been found to be an independent risk factor for the development of abdominal adiposity. ${ }^{81}$ Hypogonadism is also associated with metabolic syndrome and type 2 diabetes. ${ }^{82}$ Our KS study series demonstrated a significant decline in serum $\mathrm{T}$ by $30 \%-35 \%(P<0.01)$ at $1-12$ months, which returned to $75 \%$ of the pre-TESE levels after 18 months. The change in serum T levels in KS may have been a result of much smaller testes and Leydig cell loss near the scars after the procedures. ${ }^{83}$

In addition, Ramasamy et al. ${ }^{35}$ showed that for NOA patients, mean FSH levels increased significantly after micro-TESE, but no significant difference in LH levels was noted between the preoperative and postoperative groups. In our data, in 46XY males with NOA, serum levels of FSH increased significantly during the 18 months of follow-up after micro-TESE, and serum levels of LH at 1-3 months after micro-TESE were significantly increased from baseline concentrations, although no significant differences were observed in the levels of LH after 6 months, compared to baseline. ${ }^{83}$ Interestingly, FSH and LH concentrations in patients with KS were not significantly changed. The change in $\mathrm{FSH}$ levels for 46XY males with NOA may have been a result of local germ cell loss near the scars after the procedures. ${ }^{84}$ Tash and Schlegel ${ }^{84}$ also suggested an increase in peritubular scar tissue that may have affected Leydig cells, as well as germ cell numbers. Changes in FSH and LH levels after micro-TESE may suggest that the testes of NOA patients in general may be better able to respond than those of KS patients.

$\mathrm{T}$ itself may have a central or permissive role in the pathogenesis of metabolic syndrome and type 2 diabetes by increasing skeletal muscle tissue and decreasing abdominal obesity and non-esterified fatty acids, thus improving insulin sensitivity. ${ }^{85}$ Hypogonadism in KS may cause an unfavourable change in body composition and metabolic syndrome. ${ }^{86}$ We recommend that KS patients with low $\mathrm{T}$ levels after micro-TESE be treated properly to prevent the long-term deleterious 
consequences of hypogonadism. Many KS patients (before microTESE) were already candidates for androgen replacement based on clinical features, such as elevated LH and low-normal serum T.

Although the microdissection procedure is relatively safer and improves the sperm retrieval rate significantly in patients with NOA, we should be careful of hypogonadism in KS patients, after even microdissection procedures.

\section{ICSI OUTCOMES}

Only a few studies are available concerning the influence of retrieval techniques. In general, no differences were observed on fertilisation, implantation and clinical pregnancy rates with regard to the techniques used for NOA. ICSI cycles with testicular spermatozoa are less successful in NOA compared with OA. ${ }^{87}$ Our data also show that the overall embryo transfer rate and pregnancy rate per cycle were lower in the NOA group than in patients with normal spermatogenesis and $\mathrm{OA}$, but the pregnancy rate per cycle was not different. ${ }^{88}$ In fact, the $2 \mathrm{PN}$ fertilisation rate, the cleavage rate, the number of live births per embryo transfer and the implantation rates were not significantly different in patients with NOA than in patients with OA. In addition, using testicular sperm, no differences were noted in fertilisation or clinical pregnancy rates following cryopreservation. Thus, the results of ICSI in couples with azoospermia requiring testicular sperm retrieval indicate that the source of the spermatozoa appeared to have little effect on the prognosis.

\section{COMPETING FINANCIAL INTERESTS}

The author declares no competing financial interests.

1 Willott GM. Frequency of azoospermia. Forensic Sci Int 1982; 20: 9-10.

2 Jarow JP, Espeland MA, Lipshultz LI. Evaluation of the azoospermic patients. J Urol 1989; 142: 62

3 Temple-Smith PD, Southwick GJ, Yates CA, Trounson AO, de Kretser DM. Human pregnancy by in vitro fertilization (IVF) using sperm aspirated from the epididymis. $\mathrm{J}$ In Vitro Fert Embryo Transf 1985; 2: 119-22.

4 Schoysman R, Vanderzwalmen P, Nijs M, Segal L, Segal-Bertin G et al. Pregnancy after fertilisation with human testicular spermatozoa. Lancet 1993; 342: 1237.

5 Palermo GD, Schlegel PN, Hariprashad JJ, Ergün B, Mielnik A et al. Fertilization and pregnancy outcome with intracytoplasmic sperm injection for azoospermic men. Hum Reprod 1999; 14: 741-8.

6 Ezeh UI, Taub NA, Moore HD, Cooke ID. Establishment of predictive variables associated with testicular sperm retrieval in men with non-obstructive azoospermia. Hum Reprod 1999; 14: 1005-12.

7 Raman JD, Schlegel P. Testicular sperm extraction with intracytoplasmic sperm injection is successful for the treatment of nonobstructive azoospermia associated with cryptorchidism. J Urol 2003; 170: 1287-90.

8 Levin HS. Testicular biopsy in the study of male infertility. Hum Pathol 1979; 10 : 569-84.

9 Devroey P, Liu J, Nagy Z, Goossens A, Tournaye H et al. Pregnancies alter testicular sperm extraction and intracytoplasmic sperm injection in non-obstructive azoospermia. Hum Reprod 1995; 10: 1457-60.

10 Gil-Salom M, Minguez Y, Rubio C, de los Santos MJ, Remohi J et al. Efficacy of intracytoplasmic sperm injection using testicular spermatozoa. Hum Reprod 1995; 10: 3166-70.

11 Hauser R, Botchan A, Amit A, Ben Yosef D, Gamzu R et al. Multiple testicular sampling in non-obstructive azoospermia-is it necessary? Hum Reprod 1998; 13: 3081-5.

12 Lewin A, Weiss DB, Friedler S, Ben-Shachar I, Porat-Katz A et al. Delivery following intracytoplasmic injection of mature sperm cells recovered by testicular fine needle aspiration in a case of hypergonadotropic azoospermia due to maturation arrest. Hum Reprod 1996; 11: 769-71.

13 Fasouliotis SJ, Safran A, Porat-Katz A, Simon A, Laufer N et al. A high predictive value of the first testicular fine needle aspiration in patients with non-obstructive azoospermia for sperm recovery at the subsequent attempt. Hum Reprod 2002; 17: 139-42.

14 Turek P, Givens CR, Schriock ED, Merg MV, Pedersen RA et al. Testis sperm extraction and intracytoplasmic sperm injection guided by prior fine-needle aspiration mapping in patients with nonobstructive azoospermia. Fertil Steril 1999; 71: 552-7.

15 Hovatta O, Moilanen J, von Smitten K, Reima I. Testicular needle biopsy, open biopsy, epididymal aspiration and intracytoplasmic sperm injection in obstructive azoospermia. Hum Reprod 1995; 10: 2595-9.
16 Rajfer J, Binder S. Use of biopty gun for transcutaneous testicular biopsies. J Urol 1989; 142: 1021-2.

17 Tuuri T, Moilanen J, Kaukoranta S, Makinen S, Kotola S et al. Testicular biopty gun needle biopsy in collecting spermatozoa for intracytoplasmic injection, cryopreservation and histology. Hum Reprod 1999; 14: 1274-8.

18 Craft I, Tsirigotis M, Courtauld E, Farrer-Brown G. Testicular needle aspiration as an alternative to biopsy for the assessment of spermatogenesis. Hum Reprod 1997; 12: 1483-7.

19 Donoso P, Tournaye H, Devroey P. Which is the best sperm retrieval technique for nonobstructive azoospermia? A systematic review. Hum Reprod Update 2007; 13: 53949.

20 Rosenlund B, Kvist U, Ploen L, Lundh Rozell B, Sjoblom P et al. A comparison between open and percutaneous needle biopsies in men with azoospermia. Hum Reprod 1998; 13: 1266-71.

21 Carpi A, Menchini Fabris FG, Todeschini G, Nardini V. Large-needle percutaneous aspiration biopsy of the testicle in men with non obstructive azoospermia. Fertil Steril 2006; 86: 464-5.

22 Hauser R, Yogev L, Paz G, Yavetz H, Azem F et al. Comparison of efficacy of two techniques for testicular sperm retrieval in nonobstructive azoospermia: multifocal testicular sperm extraction versus multifocal testicular sperm aspiration. J Androl 2006; 27: 28-33.

23 Hota D, Sasturkar S. Comparison of systematic fine needle aspiration mapping and multiple microbiopsies of testis in evaluation of azoospermia. Urology 2006; 68: 61 .

24 Tournaye $\mathrm{H}$. Surgical sperm recovery for intracytoplasmic sperm injection: which method is to be preferred? Hum Reprod 1999; 14: 71-81.

25 Friedler S, Raziel A, Strassburger D, Soffer Y, Komarovsky D et al. Testicular sperm retrieval by percutaneous fine needle sperm aspiration compared with testicular sperm extraction by open biopsy in men with non-obstructive azoospermia. Hum Reprod 1997; 12: 1488-93.

26 Ostad M, Liotta D, Ye Z, Schlegel PN. Testicular sperm extraction for nonobstructive azoospermia: results of a multibiopsy approach with optimized tissue dispersion. Urology 1998; 52: 692-6.

27 Ezeh UI, Moore HD, Cooke ID. A prospective study of multiple needle biopsies versus a single open biopsy for testicular sperm extraction in men with non-obstructive azoospermia. Hum Reprod 1998; 13: 3075-80.

28 Tournaye H, Camus M, Goossens A, Liu J, Nagy P et al. Recent concepts in the management of infertility because of non-obstructive azoospermia. Hum Reprod 1995; 10: 115-9.

29 Tournaye H, Liu J, Nagy PZ, Camus M, Goossens A et al. Correlation between testicular histology and outcome after intracytoplasmic sperm injection using testicular spermatozoa. Hum Reprod 1996; 11: 127-32.

30 Amer M, El Haggar S, Moustafa T, Abd El-Naser T, Zohdy W. Testicular sperm extraction: impact of testicular histology on outcome, number of biopsies to be performed and optimal time for repetition. Hum Reprod 1999; 14: 3030-4.

31 Witt MA, Richard JR, Smith SE, Rhee EH, Tucker MJ. The benefit of additional biopsy sites when performing testicular sperm extraction in non-obstructive azoospermia. Fertil Steril 1997; 67: S79-80.

32 Schlegel PN. Testicular sperm extraction: microdissection improves sperm yield with minimal tissue excision. Hum Reprod 1999; 14: 131-5.

33 Okada H, Dobashi M, Yamazaki T, Hara I, Fujisawa M et al. Conventional versus microdissection testicular sperm extraction for nonobstructive azoospermia. J Urol 2002; 168: 1063-7.

34 Tsujimura A, Matsumiya K, Miyagawa Y, Tohda A, Miura H et al. Conventional multiple or microdissection testicular sperm extraction a comparative study. Hum Reprod 2002; 17: 2924-9.

35 Ramasamy R, Yagan N, Schlegel PN. Structural and functional changes to the testis after conventional versus microdissection testicular sperm extraction. Urology 2005; 65: 1190-4.

36 Amer M, Ateyah A, Hany R, Zohdy W. Prospective comparative study between microsurgical and conventional testicular sperm extraction in non-obstructive azoospermia follow-up by serial ultrasound examinations. Hum Reprod 2000; 15: 653-6.

37 Tsujimura A, Matsumiya K, Miyagawa Y, Takao T, Fujita K et al. Prediction of successful outcome of microdissection testicular sperm extraction in men with idiopathic nonobstructive azoospermia. J Urol 2004; 172: 1944-7.

38 Mulhall JP, Ghaly SW, Aviv N, Ahmed A. The utility of optical loupe magnification for testis sperm extraction in men with nonobstructive azoospermia. J Androl 2005; 26: 178-81.

39 Ramasamy R, Lin K, Veeck Gosden L, Rosenwaks Z, Palermo GD et al. High serum FSH levels in men with nonobstructive azoospermia does not affect success of microdissection testicular sperm extraction. Fertil Steril 2009; 92: 590-3.

40 Ishikawa T, Nose R, Yamaguchi K, Chiba K, Fujisawa M. Learning curves of microdissection testicular sperm extraction for non-obstructive azoospermia. Fertil Steril 2010; 94: 1008-11.

41 Schlegel PN, Su LM. Physiological consequences of testicular sperm extraction. Hum Reprod 1997; 12: 1688-92.

42 Friedler S, Raziel A, Schachter M, Strassburger D, Bern 0 et al. Outcome of first and repeated testicular sperm extraction and ICSI in patients with non-obstructive azoospermia. Hum Reprod 2002; 17: 2356-61.

43 Tsujimura A, Miyagawa Y, Takao T, Takada S, Koga M et al. Salvage microdissection testicular sperm extraction after failed conventional testicular sperm extraction in patients with nonobstructive azoospermia. J Urol 2006; 175: 1446-9. 
44 Ramasamy R, Schlegel PN. Microdissection testicular sperm extraction: effect of prior biopsy on success of sperm retrieval. J Urol 2007; 177: 1447-9.

45 Bojesen A, Juul S, Gravholt CH. Prenatal and postnatal prevalence of Klinefelter syndrome: a national registry study. J Clin Endocrinol Metab 2003; 88: 622-6.

46 Tournaye H, Staessen C, Liebaers I, van Assche E, Devroey P et al. Testicular sperm recovery in nine 47,XXY Klinefelter patients. Hum Reprod 1996; 11: 1644-9.

47 Reubinoff BE, Abeliovich D, Werner M, Schenker JG, Safran A et al. A birth in nonmosaic Klinefelter's syndrome after testicular fine needle aspiration, intracytoplasmic sperm injection and preimplantation genetic diagnosis. Hum Reprod 1998; 13: 1887-92.

48 Okada H, Goda K, Yamamoto Y, Sofikitis N, Miyagawa I et al. Age as a limiting factor for successful sperm retrieval in patients with nonmosaic Klinefelter's syndrome. Ferti Steril 2005; 84: 1662-4.

49 Schiff JD, Palermo GD, Veeck LL, Goldstein M, Rosenwaks Z et al. Success of testicular sperm injection and intracytoplasmic sperm injection in men with Klinefelter syndrome. J Clin Endocrinol Metab 2005; 90: 6263-7.

50 Koga M, Tsujimura A, Takeyama M, Kiuchi H, Takao T et al. Clinical comparison of successful and failed microdissection TESE in patients with non-mosaic Klinefelter syndrome. Urology 2007; 70: 341-5.

51 Vernaeve V, Staessen C, Verheyen G, van Steirteghem A, Devroey P et al. Can biological or clinical parameters predict testicular sperm recovery in $47 \mathrm{XXY}$ Klinefelter's syndrome patients? Hum Reprod 2004; 19: 1135-9.

52 Emre Bakircioglu M, Erden HF, Kaplancan T, Ciray N, Bener F et al. Aging may adversely affect testicular sperm recovery in patients with Klinefelter syndrome. Urology 2006; 68: 1082-6

53 Ramasamy R, Ricci JA, Palermo GD, Gosden LV, Rosenwaks Z et al. Successful fertility treatment for Klinefelter's syndrome. J Urol 2009; 182: 1108-13.

54 Har-Toov J, Eytan O, Hauser R, Yavetz H, Elad D et al. A new power Doppler ultrasound guiding technique for improved testicular sperm extraction. Fertil Steril 2004; 81 430-4.

55 Herwig R, Tosun K, Schuster A, Rehder P, Glodny B et al. Tissue perfusion-controlled guided biopsies are essential for the outcome of testicular sperm extraction. Ferti Steril 2007; 87: 1071-6.

56 Tournaye H, Verheyen G, Nagy P, Ubaldi F, Goossens A et al. Are there any predictive factors for successful testicular sperm recovery in azoospermic patients? Hum Reprod 1997; 12: 80-6

57 Seo JT, Ko WJ. Predictive factors of successful testicular sperm recovery in nonobstructive azoospermia patients. Int J Androl 2001; 24: 306-10.

58 Anniballo R, Ubaldi F, Cobellis L, Sorrentino M, Rienzi L et al. Criteria predicting the absence of spermatozoa in the Sertoli cell-only syndrome can be used to improve success rates of sperm retrieval. Hum Reprod 2000; 15: 2269-77.

59 Silber S, Nagy Z, Devroey P, Tournaye H, van Steirteghem AC. Distribution of spermatogenesis in the testicles of azoospermic men: the presence or absence of spermatids in the testes of men with germinal failure. Hum Reprod 1997; 12 2422-8.

60 Brandell RA, Mielnik A, Liotta D, Ye Z, Veeck LL et al. AZFb deletions predict the absence of spermatozoa with testicular sperm extraction: preliminary report of a prognostic genetic test. Hum Reprod 1998; 13: 2812-5.

61 Gil-Salom M, Romero J, Minguez Y, Molero MD, Remohi J et al. Testicular sperm extraction and intracytoplasmic sperm injection: a chance of fertility in nonobstructive azoospermia. J Urol 1998; 160: 2063-7.

62 Krausz C, Quinatana-Murci L, Mcelreavey K. Prognostic value of $Y$ deletion analysis: what is the clinical prognostic value of $Y$ chromosome microdeletion analysis? Hum Reprod 2000; 15: 1431-4.

63 Bromage SJ, Falconer DA, Lieberman BA, Sangar V, Payne SR. Sperm retrieval rates in subgroups of primary azoospermic males. Eur Urol 2007; 51: 534-9.

64 Chen CS, Chu SH, Lai YM, Wang ML, Chan PR. Reconsideration of testicular biopsy and follicle-stimulating hormone measurement in the era of intracytoplasmic sperm injection for nonobstructive azoospermia? Hum Reprod 1996; 11: 2176-9.

65 Ezeh UI, Taub NA, Moore HD, Cooke ID. Establishment of predictive variables associated with testicular sperm retrieval in men with nonobstructive azoospermia. Hum Reprod 1999; 14: 1005-12.

66 Jezek D, Knuth UA, Schulze W. Successful testicular sperm extraction (TESE) in spite of high serum follicle stimulating hormone and azoospermia: correlation between testicular morphology, TESE results, semen analysis and serum hormone values in 103 infertile men. Hum Reprod 1998; 13: 1230-4.
67 Kahraman S, Ozgür S, Alataș C, Aksoy S, Balaban B et al. High implantation and pregnancy rates with testicular sperm extraction and intracytoplasmic sperm injection in obstructive and nonobstructive azoospermia. Hum Reprod 1996; 11 673-6.

68 Silber SJ, van Steirteghem A, Nagy Z, Liu J, Tournaye H et al. Normal pregnancies resulting from testicular sperm extraction and intracytoplasmic sperm injection for azoospermia due to maturation arrest. Fertil Steril 1996; 66: 110-7.

69 von Eckardstein S, Simoni M, Bergmann M, Weinbauer GF, Gassner P et al Serum inhibin B in combination with serum follicle-stimulating hormone (FSH) is a more sensitive marker than serum FSH alone for impaired spermatogenesis in men, but cannot predict the presence of sperm in testicular tissue samples. J Clin Endocrino Metab 1999; 84: 2496-501.

70 Foresta C, Bettella A, Petraglia F, Pistorello M, Luisi S et al. Inhibin B levels in azoospermic subjects with cytologically characterized testicular pathology. Clin Endocrinol (Oxf) 1999; 50: 695-701.

71 Vernaeve V, Tournaye H, Schiettecatte J, Verheyen G, van Steirteghem A et al. Serum inhibin $B$ cannot predict testicular sperm retrieval in patients with non-obstructive azoospermia. Hum Reprod 2002; 17: 971-6.

72 Tunc L, Kirac M, Gurocak S, Yucel A, Kupeli B et al. Can serum inhibin B and FSH levels, testicular histology and volume predict the outcome of testicular sperm extraction in patients with non-obstructive azoospermia? Int Urol Nephrol 2006; 38: 629-35.

73 Mulhall JP, Burgess CM, Cunningham D, Carson R, Harris D et al. Presence of mature sperm in testicular parenchyma of men with nonobstructive azoospermia: prevalence and predictive factors. Urology 1997; 49: 91-5.

74 Ramasamy R, Ricci JA, Leung RA, Schlegel PN. Successful repeat microdissection testicular sperm extraction in men with nonobstructive azoospermia. J Urol 2011; 185: 1027-31.

75 Johnson L. Evaluation of the human testis and its age-related dysfunction. Prog Clin Biol Res 1989; 302: 35-60.

76 Ramasamy R, Fisher ES, Ricci JA, Leung RA, Schlegel PN. Duration of microdissection testicular sperm extraction procedures: relationship to sperm retrieval success. J Urol 2011; 185: 1394-7.

77 Ron-El R, Strauss S, Friedler S, Strassburger D, Komarovsky D et al. Serial sonography and colour Doppler imaging following testicular and epididymal sperm extraction. Hum Reprod 1998; 13: 3390-3.

78 Silber SJ. Microsurgical TESE and the distribution of spermatogenesis in nonobstructive azoospermia. Hum Reprod 2000; 15: 2278-84.

79 Harrington TG, Schauer D, Gilbert BR. Percutaneous testis biopsy: an alternative to open testicular biopsy in the evaluation of the subfertile man. J Urol 1996; 156: 1647-51.

80 Komori K, Tsujimura A, Miura H, Shin M, Takada T et al. Serial follow-up study of serum testosterone and antisperm antibodies in patients with non-obstructive azoospermia after conventional or microdissection testicular sperm extraction. Int Androl 2004; 27: 32-6.

81 van den Bergh JP, Hermus AR, Spruyt AI, Sweep CG, Corstens FH et al. Bone minera density and quantitative ultrasound parameters in patients with Klinefelter's syndrome after long-term testosterone substitution. Osteop Int 2001; 12: 55-62.

82 Horowitz M, Wishart JM, O'Loughlin PD, Morris HA, Need AG et al. Osteoporosis and Klinefelter's syndrome. Clin Endocrinol (Oxf) 1992; 36: 1138

83 Ishikawa T, Yamaguchi K, Chiba K, Takenaka A, Fujisawa M. The assessment of serum hormone levels in patients with non-obstructive azoospermia after microdissection testicular sperm extraction. J Urol 2009; 182: 1495-9.

84 Tash J, Schlegel PN. The histologic effects of TESE on the testicle in men with nonobstructive azoospermia. Urology 2001; 57: 334-7.

85 Mårin $\mathrm{P}$, Holmäng S, Jönsson L, Sjöström L, Kvist $\mathrm{H}$ et al. The effects of testosterone treatment on body composition and metabolism in middle-aged obese men. Int J Obes Relat Metab Disord 1992; 16: 991-7.

86 Ishikawa T, Yamaguchi K, Kondo Y, Takenaka A, Fujisawa M. Metabolic syndrome in men with Klinefelter syndrome. Urology 2008; 71: 1109-13.

87 Nicopoullos J, Gilling-Smith C, Almeida P, Norman-Taylor J, Grace I et al. Use of surgical sperm retrieval in azoospermic men: a meta-analysis. Fertil Steril 2004; 82: 691-700.

88 Ishikawa T, Shiotani M, Izumi Y, Hashimoto H, Kokeguchi S et al. Fertilization and pregnancy using cryopreserved testicular sperm for intracytoplasmic sperm injection with azoospermia. Fertil Steril 2009; 92: 174-9. 